\title{
Conditional convergence of photorefractive perceptron learning
}

\author{
Ken Y. Hsu, Shiuan Huei Lin, and Pochi Yeh* \\ Institute of Electro-Optic Engineering, National Chiao Tung University, Hsinchu, Taiwan, China
}

Received August 6, 1993

\begin{abstract}
We consider the convergence characteristics of a perceptron learning algorithm, taking into account the decay of photorefractive holograms during the process of interconnection weight changes. As a result of the hologram erasure, the convergence of the learning process is dependent on the exposure time during the weight changes. A mathematical proof of the conditional convergence, as well as computer simulations of the photorefractive perceptrons, is presented and discussed.
\end{abstract}

It is well known that the iterations in the perceptron learning algorithm will converge, leading to a final weight vector (or matrix), provided that such a solution exists. ${ }^{1}$ Recently the perceptron learning algorithm was implemented by optical interconnection with photorefractive holograms. ${ }^{2-5}$ In the optical implementation, the weight vector $w$ is represented by the holograms in photorefractive crystals (e.g., $\mathrm{LiNbO}_{3}$ ). The weight vector can be modified by addition or subtraction of hologram components according to the learning algorithm. Holograms previously stored in photorefractive media are subject to erasure during the training cycles. Thus the perceptron learning algorithm in photorefractive media is modified as

$$
\begin{aligned}
\mathbf{w}(k+1)= & \mathbf{w}(k) \exp [-|\sigma(k)| t / \tau] \\
& +\sigma(k)[1-\exp (-t / \tau)] \cdot \mathbf{x}(k),
\end{aligned}
$$

where $t$ is the exposure time, $\tau$ is the hologram decay time constant, $k$ is an integer registering the number of interrogations, $\mathbf{x}(k)$ is the $k$ th input vector, and $\sigma(k)$ is given by

$$
\sigma(k)=\left\{\begin{aligned}
0 & \text { if } \mathbf{x}(k) \text { is correctly classified } \\
1 & \text { if } \mathbf{x}(k) \in C 1 \text { but } \mathbf{w}(k) \cdot \mathbf{x}(k)<\theta, \\
-1 & \text { if } \mathbf{x}(k) \in C 2 \text { but } \mathbf{w}(k) \cdot \mathbf{x}(k)>\theta
\end{aligned}\right.
$$

where $\theta$ is the threshold value and $C 1$ (or $C 2$ ) represents the category of the patterns whose outputs are 1 (or -1 ).

It is interesting to note that the existing interconnection weight $\mathbf{w}(k)$ is reduced by a hologram decay factor $\exp (-t / \tau)$ because of the illumination of light during each of the weight changes. It is clear that the exposure time $t$ plays an important role in determining the magnitude of the incremental weight change as well as the decay of the weight vector. Although there have been several previous studies in the implementation of perceptron in photorefractive media, there is to our knowledge no theoretical groundwork on the issue of convergence. In this Letter we consider the convergence characteristics of the photorefractive perceptron and present what is to our knowledge the first theoretical proof of the conditional convergence. We discover that the photorefractive perceptron learning algorithm according to Eq. (1) will converge, provided that the exposure time is sufficiently small relative to the hologram decay time constant. In addition to the theoretical proof of the conditional convergence, we also present and discuss the results of our computer simulation.

To prove the conditional convergence, we redefine the set of training patterns as

$$
\mathbf{y}=\left\{\begin{array}{rl}
\mathbf{x} & \mathbf{x} \in C 1 \\
-\mathbf{x} & \mathbf{x} \in C 2
\end{array}\right.
$$

With this definition, the training algorithm of Eq. (1) becomes

$\mathbf{w}(p+1)=\mathbf{w}(p) \exp (-t / \tau)+[1-\exp (-t / \tau)] \cdot \mathbf{y}(p)$.

We note that $p$ is an integer registering the number of weight changes. Assuming that the initial condition is 0, i.e., $\mathbf{w}(1)=0$, we obtain, according to Eq. (4),

$$
\mathbf{w}(p+1)=[1-\exp (-t / \tau)] \sum_{k=1}^{p} \mathbf{y}(k) \exp [-(p-k) t / \tau]
$$

Following the analysis used in the convergence proof of the conventional perceptron ${ }^{6}$ and carrying out the summation of the exponential terms, we obtain

$$
|\mathbf{w}(p+1)|^{2}>\frac{[1-\exp (-p t / \tau)]^{2} \cdot \beta^{2}}{|\mathbf{w}|^{2}},
$$

where we have assumed that there exists a solution $\mathbf{w}$ and that $\beta$ is given by

$$
\beta=\min _{1 \leq k \leq M} \mathbf{w} \cdot \mathbf{y}(k) .
$$

Using $\mathbf{w}(1)=0$ and following similar steps, we also obtain

$$
|\mathbf{w}(p+1)|^{2}<\frac{[1-\exp (-t / \tau)]^{2} \cdot \alpha \cdot[1-\exp (-2 p t / \tau)]}{[1-\exp (-2 t / \tau)]}
$$


where

$$
\alpha=\max _{1 \leq k \leq M}|\mathbf{y}(k)|^{2}
$$

Combining Eqs. (6) and (8), we obtain the following condition for the number of iterations $p$ :

$$
\frac{\tanh (p t / 2 \tau)}{\tanh (t / 2 \tau)}<\frac{\alpha|\mathbf{w}|^{2}}{\beta^{2}} \equiv P_{i \max },
$$

where $P_{i \max }$ is the upper bound of the number of weight changes to reach convergence for the conventional perceptron. In practice, it is not easy for one to determine $P_{i \max }$ from analytic solutions since there are infinite numbers of $\mathbf{w}$. Using the training set and the exposure time, we can obtain only a rough range of the minimum of $P_{i \max }$ by computer simulation. It provides a guideline for determining the proper exposure time for optical implementations.

Before the discussion of the convergence, let us first understand the meaning of inequality (10). We recall that the weight is modified only when $\mathbf{w} \cdot \mathbf{y}<0$ (i.e., when misclassification occurs). Based on the definition, the total number of weight changes will reach a definite value $p$ when the system is fully trained. This number $p$ must satisfy inequality (10). If we can find some number $p_{0}$, from a purely mathematical point of view, such that

$$
\frac{\tanh \left(p_{0} t / 2 \tau\right)}{\tanh (t / 2 \tau)}=\frac{\alpha|\mathbf{w}|^{2}}{\beta^{2}}=P_{i \max },
$$

then $p_{0}$ is the upper bound for the total number of weight changes. When either the hologram decay time constant is very long or the exposure time is very short, i.e., $t / 2 \tau$ is near zero, inequality (10) reduces to the well-known result of the conventional perceptron,

$$
p<P_{i \max }
$$

In this case, $P_{i \max }$ is the upper bound for the total number of weight changes.

In Fig. 1 we plot both sides of inequalities (10) and (12) as a function of $p$. For inequality (10) the lefthand side is a hyperbolic-tangent function, and for inequality (12) it is a straight line at $45^{\circ}(y=p)$. The right-hand side of each formula is a horizontal line at $y=P_{i \max }$. For the case of a conventional perceptron, this line will always intersect the $45^{\circ}$ line at $P_{i \max }$. For the case of a photorefractive perceptron, the intersection exists only when

$$
\tanh (t / 2 \tau) P_{i \max }<1 .
$$

For $t / 2 \tau \ll 1$, the condition becomes

$$
(t / 2 \tau) P_{i \max }<1
$$

In other words, the photorefractive perceptron will converge, provided that $\tanh (t / 2 \tau) P_{i \max }<1$. Let the intersection be at $p_{0}$; we find that the maximum number of steps for convergence is greater than that for the conventional perceptron (i.e., $P_{i \max }<p_{0}$ ). This is consistent with the fact that the holograms are decaying during the weight changes. It will take more steps (or training cycles) to achieve the solution w. When the exposure time is too long, such that

$$
(t / 2 \tau) P_{i \max }>1
$$

Eq. (11) has no solution. In other words, there is no upper bound for the number of weight changes, and the photorefractive perceptron learning algorithm may not converge to a solution.

We now discuss the results of our computer simulation. Figure 2 shows our set of training patterns. The three roman letters are specified as class $C 1$, and the three arabic numbers are specified as class $C 2$. We first train the classifier by using the conventional perceptron algorithm. For the input vectors shown in Fig. 2, it takes three training cycles with six weight changes (i.e., $p=6$ ) for the training processing to be completed. The simulation results indicate that these six training patterns are linearly separable. We then simulate the photorefractive perceptron according to the learning algorithm given by Eq. (1). The simulation results are shown in Fig. 3. The figure shows the number of weight changes $p$ as well as the number of training cycles as functions of the normalized exposure time $t / \tau$. We note that when $t / \tau \ll 1$ the number of weight changes and the number of training cycles are nearly constant and are identical to those of the conventional perceptron. This confirms our previous statement that the convergence behavior is similar to that of the conventional perceptron if the hologram decay is

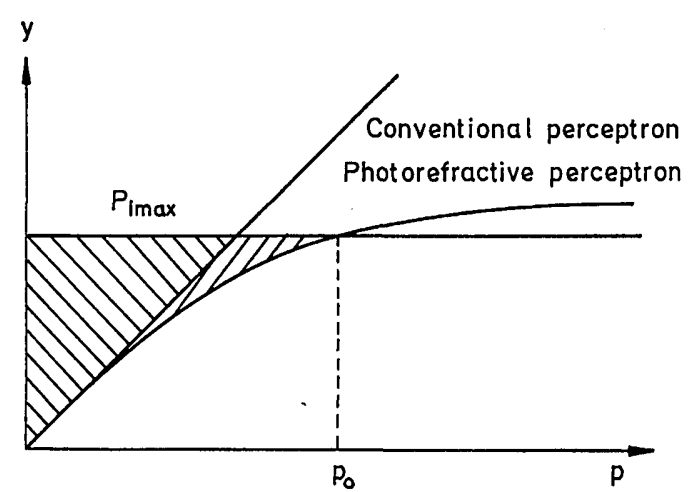

Fig. 1. Graphic method for finding solutions of inequality (10). Intersection occurs only when the condition of inequality (13) is satisfied.
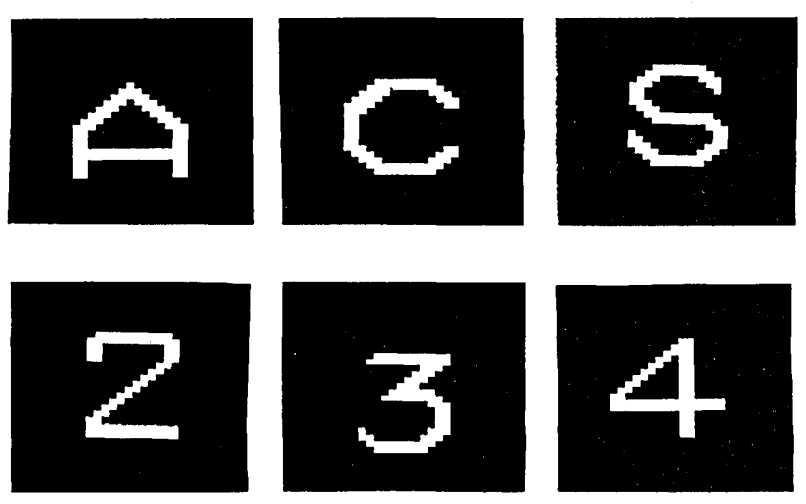

Fig. 2. Pictures of our training patterns. The sampling grid of the computer simulation is $32 \times 32$. 


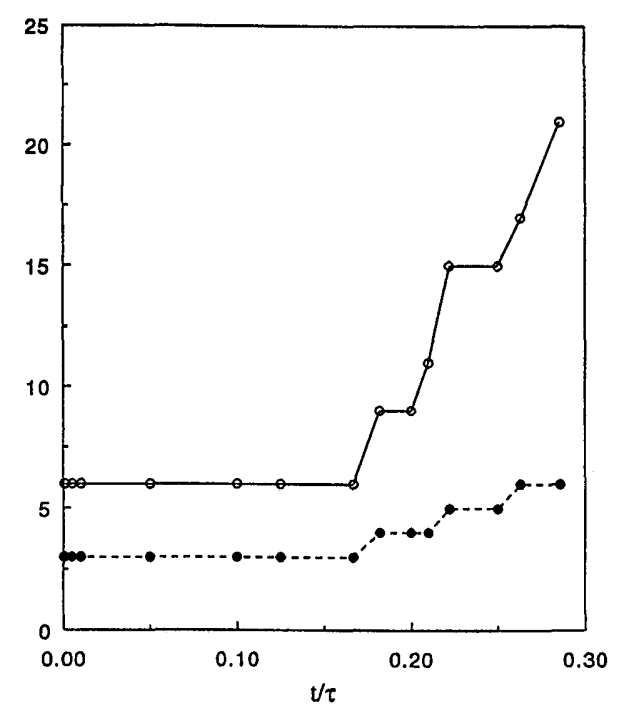

Fig. 3. Number of steps (open circles, number of weight changes; filled circles, number of training cycles) for photorefractive perceptron convergence as functions of the normalized exposure time $t / \tau$.

Table 1. Computer Simulation of Photorefractive Perceptrons with Different $t / \tau$ Values

\begin{tabular}{lccc}
\hline \multicolumn{1}{c}{$t / \tau$} & $P_{i \max }$ & $P_{i \max } \tanh (t / 2 \tau)$ & $\begin{array}{c}\text { No. of Weight } \\
\text { Changes }\end{array}$ \\
\hline 0.0005 & 37.01 & $9.25 \times 10^{-3}$ & 6 \\
0.001 & 37.28 & $1.86 \times 10^{-2}$ & 6 \\
0.005 & 39.51 & $9.88 \times 10^{-2}$ & 6 \\
0.01 & 42.56 & 0.213 & 6 \\
0.05 & 84.88 & 2.12 & 6 \\
0.1 & 300.07 & 14.991 & 6 \\
0.125 & 830.27 & 51.824 & 6 \\
0.167 & $8.1 \times 10^{8}$ & $6.75 \times 10^{7}$ & 6 \\
0.182 & 3011.2 & 273.265 & 9 \\
0.2 & $6.7 \times 10^{7}$ & $6.68 \times 10^{3}$ & 9 \\
0.21 & 79 & 8.265 & 11 \\
0.222 & 874.2 & 96.64 & 15 \\
0.25 & $5.15 \times 10^{4}$ & $6.4 \times 10^{3}$ & 15 \\
0.263 & $2.79 \times 10^{4}$ & $3.65 \times 10^{3}$ & 17 \\
0.286 & 367.5 & 52.197 & 21 \\
0.333 & - & - & - \\
0.5 & - & - & - \\
\hline
\end{tabular}

negligible. We also note that when the normalized exposure time $t / \tau$ is greater than 0.16 the number of steps for convergence increases significantly. This is in agreement with the fact that the photorefractive perceptron will need to take more steps (training cycles) to converge in order to overcome the decay of the holograms. In the simulation when the normalized exposure time $t / \tau$ is greater than 0.3 , the error rate remains $100 \%$ for all training cycles, and the system does not converge. Again, this is in agreement with our earlier prediction that the perceptron may not converge when the exposure time does not satisfy inequality (13). Table 1 summarizes the results of our computer simulation for various exposure times. We note that there are many solutions for the weight vector. Each of these weight vectors corresponds to an upper bound $P_{i \max }$. In the table, $P_{i \max }$ is evaluated by the definition of inequality (10) with $\mathbf{w}=\mathbf{w}(p+1)$. The table also tests the convergence condition of inequality (10). We recall that inequality (13) gives a sufficient condition for convergence only. Violation of the condition does not constitute divergence. This explains the convergent cases in which $\tanh (t / 2 \tau) P_{i \max }>1$ in Table 1.

In summary, we have described the conditional convergence of the photorefractive perceptron. To our knowledge, this is the first mathematical proof of the conditional convergence of photorefractive perceptrons. We find that the convergence of photorefractive perceptron learning depends on the exposure time during each of the weight changes. In addition, we also provide results of our computer simulations. The simulation results are in excellent agreement with the mathematical proof.

This research is supported by a grant from the National Science Council of Taiwan under contract NSC 82-0416-E-009-195. P. Yeh acknowledges support from the K. T. Lee and K. Y. Chin Foundation. The authors are grateful for the helpful comments from one of the reviewers.

*Permanent address, Department of Electrical Engineering, University of California, Santa Barbara, Santa Barbara, California 93106.

\section{References}

1. R. O. Duda and P. E. Hart, Pattern Classification and Scene Analysis (Wiley, New York, 1973), Chap. 5, p. 142 .

2. D. Psaltis, D. Brady, and K. Wagner, Appl. Opt. 27, 1752 (1988).

3. J. Hong, S. Campbell, and P. Yeh, Appl. Opt. 29, 3019 (1990).

4. K. Y. Hsu, S. H. Lin, C. J. Cheng, T. C. Hsieh, and P. Yeh, Int. J. Opt. Comput. (to be published).

5. E. G. Paek, J. R. Wullert II, and J. S. Patel, Opt. Lett. 14, 1303 (1989).

6. See, for example, P. Yeh, Introduction to Photorefractive Nonlinear Optics (Wiley, New York, 1993), App. A, pp. 392-393. 